\title{
Genetic Algorithm Assisted Minimum Bit Error Rate Multiuser Detection in Multiple Antenna Aided OFDM
}

\author{
M. Y. Alias, S. Chen, ${ }^{1}$ L. Hanzo \\ School of ECS., Univ. of Southampton, SO17 1BJ, UK. \\ Tel: +44-23-8059 3125, Fax: +44-23-8059 3045 \\ Email: ${ }^{1} \mathrm{lh} @$ ecs.soton.ac.uk, http://www-mobile.ecs.soton.ac.uk
}

\begin{abstract}
The family of minimum bit error rate (MBER) multiuser detectors (MUD) is capable of outperforming the classic minimum mean-squared-error (MMSE) MUD in term of the achievable bit-error rate (BER) owing to directly minimising the BER cost function. In this paper, we will invoke genetic algorithms (GA) for finding the optimum weight vectors of the MBER MUD in the context of multipleantenna aided multi-user OFDM.
\end{abstract}

\section{INTRODUCTION}

In an effort to increase the achievable system capacity of an OFDM system, antenna arrays can be employed for supporting multiple users in a Space Division Multiple Access (SDMA) communications scenario $[1,2]$. A variety of linear multiuser detectors (MUD) have been proposed for performing the separation of OFDM users based on their unique, user-specific, spatial signature, provided that their channel impulse response was accurately estimated $[1,2]$. The most popular SDMAreceiver design strategy is constituted by the minimum meansquared-error (MMSE) MUD.

However, as recognised in [3-5], a better strategy is to choose the linear detector's coefficients so as to directly minimise the error-probability or bit-error ratio (BER), rather than the mean-squared error (MSE). This is because minimising the MSE does not necessarily guarantee that the BER of the system is also minimised. The family of detectors that directly minimises the BER is referred to as the minimum bit-error rate (MBER) detector class [3-6]. In [7] we have derived the exact MBER MUD weight calculation for the uplink SDMA OFDM system. We have also shown that the MBER MUD may significantly outperform the MMSE MUD in terms of the achievable BER in a two-user OFDM scenario.

In this contribution, we will investigate the performance of the proposed MBER MUD with the assistance of genetic algorithm for finding the MUD's weight vectors, as an alternative to the simplified conjugate gradient (CG) algorithm of [7].

\section{SYSTEM MODEL}

\subsection{Space Division Multiple Access (SDMA)}

Fig. 1 portrays the antenna array aided uplink transmission scenario considered. In this figure, each of the $L$ simultaneous users is equipped with a single transmission antenna, while the base-station's receiver capitalises on a $P$-element antenna front-end. The set of complex signals, $x_{p}[n, k], p \in 1, \ldots, P$ received by the $P$-element antenna array in the $k$-th subcarrier of the $n$-th OFDM symbol is constituted by the superposition of the independently faded signals associated with the $L$ users sharing the same space-frequency resource [1]. The received signal was corrupted by the Gaussian noise at the array elements. The indices $[n, k]$ have been omitted for notational convenience during our forthcoming discourse, yielding [1]:

$$
\mathbf{x}=\mathbf{H s}+\mathbf{n}=\overline{\mathbf{x}}+\mathbf{n}
$$

where $\mathbf{x}$ is the $(P \times 1)$-dimensional vector of the received signals, $\mathbf{s}$ is the $(L \times 1)$-dimensional vector of transmitted signals, $\mathbf{n}$ is the $(P \times 1)$-dimensional noise vector and $\overline{\mathbf{x}}$ represents the noiseless component of $\mathbf{x}$. The complex data signal, $s_{l}$, transmitted by the $l$-th user, $l \in 1, \ldots, L$ and the AWGN noise process, $n_{p}$, at any antenna array element $p, p \in 1, \ldots, P$ are assumed to exhibit a zero mean and a variance of $\sigma_{l}^{2}$ and $2 \sigma_{n}^{2}$ for the data signal and AWGN noise process, respectively.

The frequency domain channel transfer function (FDCHTF) matrix $\mathbf{H}$ of dimension $P \times L$ is constituted by the set of channel transfer function vectors of the $L$ users, each of which describes the FDCHTF between the single transmitter antenna associated with a particular user $l$ and the reception array elements $p \in 1, \ldots, P$. The FDCHTFs, $H_{p l}$ of the different array elements $p \in 1, \ldots, P$ for users $l \in 1, \ldots, L$ are independent, stationary, and complex Gaussian distributed processes with zero-mean and unit variance.

For the MUDs, the estimate $\hat{\mathbf{s}}$ of the transmitted signal vector s of the $L$ simultaneous users is generated by linearly combining the signals received by the $P$ different antenna elements at the BS with the aid of the array weight matrix $\mathbf{W}$, resulting in:

$$
\hat{\mathbf{s}}=\mathbf{W}^{H} \mathbf{x} .
$$




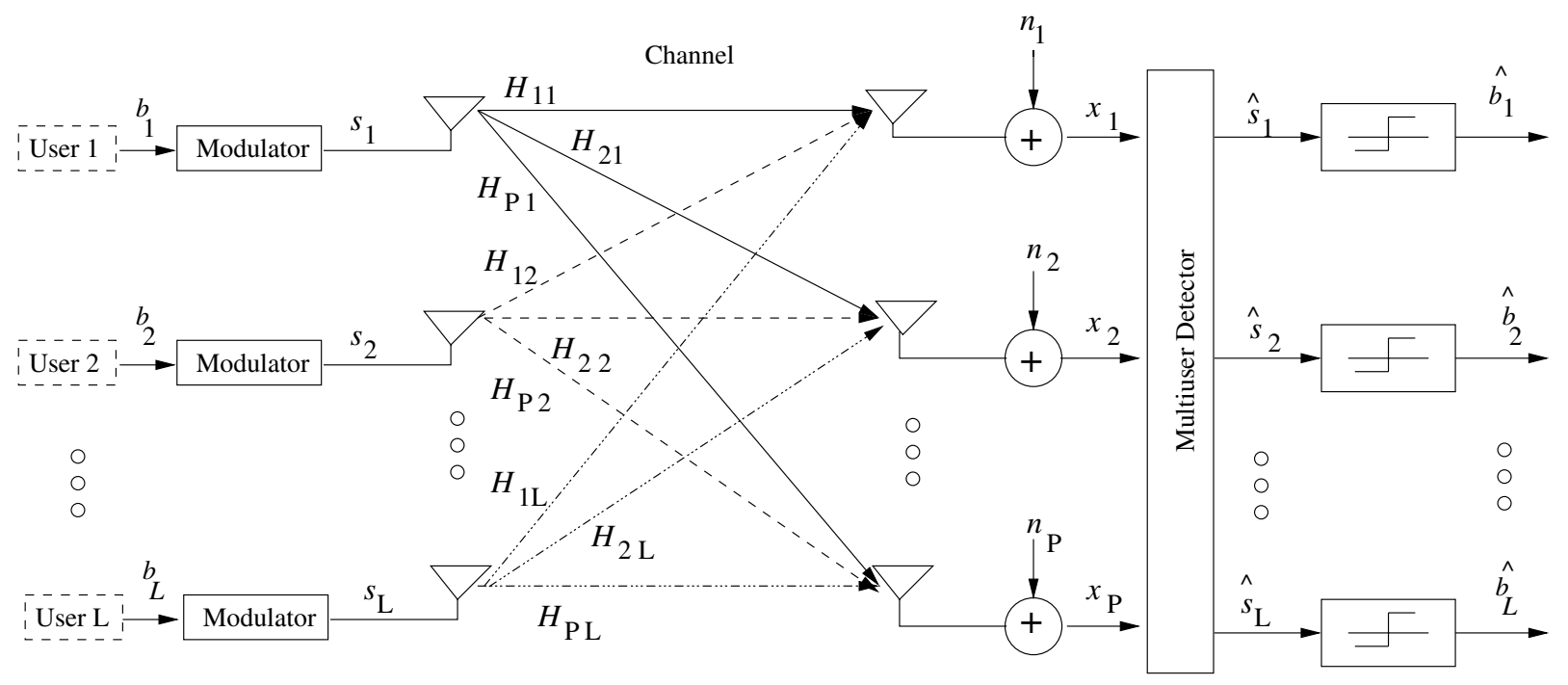

Figure 1: Schematic of an antenna array aided OFDM uplink scenario, where each of the $L$ users is equipped with a single transmit antenna and the BS's receiver is assisted by a $P$-element antenna front-end.

By substituting $\mathrm{x}$ with Eq. 1 and considering the $l$-th user's associated vector component, we will arrive at:

$$
\begin{aligned}
\hat{s}_{l} & =\mathbf{w}_{l}^{H} \mathbf{x}=\mathbf{w}_{l}^{H} \mathbf{H} \mathbf{s}+\mathbf{w}_{l}^{H} \mathbf{n} \\
& =\bar{s}_{l}+\mathbf{w}_{l}^{H} \mathbf{n},
\end{aligned}
$$

where the weight vector $\mathbf{w}_{l}$ is the $l$-th column of the weight matrix W. At the current state-of-the-art, the most popular MUD strategy is the MMSE design, where $\mathbf{w}_{l}$ is chosen as the unique vector minimising the MSE expressed as MSE = $E\left[\left(\hat{s}_{l}-s_{l}\right)^{2}\right]$, namely as [1]:

$$
\mathbf{w}_{l(M M S E)}=\left(\mathbf{H H}^{H}+2 \sigma_{n}^{2} \mathbf{I}\right)^{-1} \mathbf{H}_{l},
$$

where $\mathbf{H}_{l}$ is the $l$-th column of the FDCHTF matrix $\mathbf{H}$.

\subsection{Exact MBER Multiuser Detection}

In our initial discourse we assumed the explicit knowledge of the FDCHTF matrix $\mathbf{H}$ defined in Eq. 1. However, in practice $\mathbf{H}$ has to be determined on the basis of the channel-impaired noisy value of $\mathbf{x}$ and hence a number of adaptive techniques have been proposed in references $[4,5]$ to this effect.

The probability of error $P_{E}$ encountered at the output of the SDMA MUD characterised by the combiner weight vector $\mathbf{w}_{l}$ of user $l$ may be expressed as [7]:

$$
P_{E}\left(\mathbf{w}_{l}\right)=\frac{1}{N_{b}} \sum_{j=1}^{N_{b}} Q\left[\frac{\operatorname{sgn}\left(b_{l}^{(j)}\right) \cdot \mathbf{w}_{l}^{H} \overline{\mathbf{x}}_{j}}{\sigma_{n} \sqrt{\mathbf{w}_{l}^{H} \mathbf{w}}}\right],
$$

where $N_{b}$ is the number of equiprobable combinations of the binary vectors of the $L$ users, i.e. we have $N_{b}=2^{L}, \sigma_{n}$ is the variance of the noise, $b_{l}^{(j)} j \in 1, \ldots, N_{b}$ is the transmitted bit of user $l$, and $\overline{\mathbf{x}}_{j}, j \in 1, \ldots, N_{b}$ constitutes a possible value of the noiseles $(P \times 1)$-dimensional received signal vector $\overline{\mathbf{x}}$.

The MBER solution is defined as [5]:

$$
\mathbf{w}_{l(M B E R)}=\arg \min _{\mathbf{w}_{l}} P_{E}\left(\mathbf{w}_{l}\right) .
$$

However, the complex, irregular shape of the BER cost function prevents us from deriving a closed-form solution for the MBER MUD weights. Therefore, in practice an iterative strategy based on the steepest-descent gradient method can be used for finding the MBER solution [5]. According to this method, the linear SDMA MUD's weight vector $\mathbf{w}_{l}$ is iteratively updated, commencing for example from the MMSE weights, until the specific SDMA MUD weight vector that exhibits the lowest BER is arrived at. In each step, the weight vector is updated according to a specific step-size, $\mu$, in the vectorial direction in which the BER cost function decreases most rapidly, namely in the direction opposite to the gradient of the BER cost function, which is given by:

$$
\begin{aligned}
\nabla_{\mathbf{w}_{l}} P_{E}\left(\mathbf{w}_{l}\right)= & \frac{1}{N_{b} \sqrt{2 \pi} \sigma_{n}}\left(\frac{\mathbf{w}_{l} \mathbf{w}_{l}^{H}-\mathbf{w}_{l}^{H} \mathbf{w}_{l} \mathbf{I}}{\left(\mathbf{w}_{l}^{H} \mathbf{w}_{l}\right)^{\frac{3}{2}}}\right) \\
& \cdot \sum_{j=1}^{N_{b}} e^{\left(-\frac{\left(\bar{s}_{l}^{(j)}\right)^{2}}{2 \sigma_{n}^{2} \mathbf{w}_{l}^{H} \mathbf{w}_{l}}\right)} \cdot \operatorname{sgn}\left(b_{l}^{(j)}\right) \cdot \overline{\mathbf{x}}_{j},(7)
\end{aligned}
$$

where $\bar{s}_{l}^{(j)}=\mathbf{w}_{l}^{H} \overline{\mathbf{x}}_{j}$.

The BER is independent of the magnitude of the MUD's weight vector [7], and hence the knowledge of the orientation of the detector's weight vector is sufficient for defining the decision boundary of the linear MBER OFDM/SDMA detector. Therefore, the MBER detector has an infinite number of MUD weight solutions, although with the aid of appropriate weightvector normalisation, it is possible to reduce the infinite number of MBER solutions to a single solution.

In [7], we employed the simplified cojugate gradient (CG) algorithm for arriving at the minimum solution of the BER cost function, and the step size, $\mu$, is fixed for every iteration. 


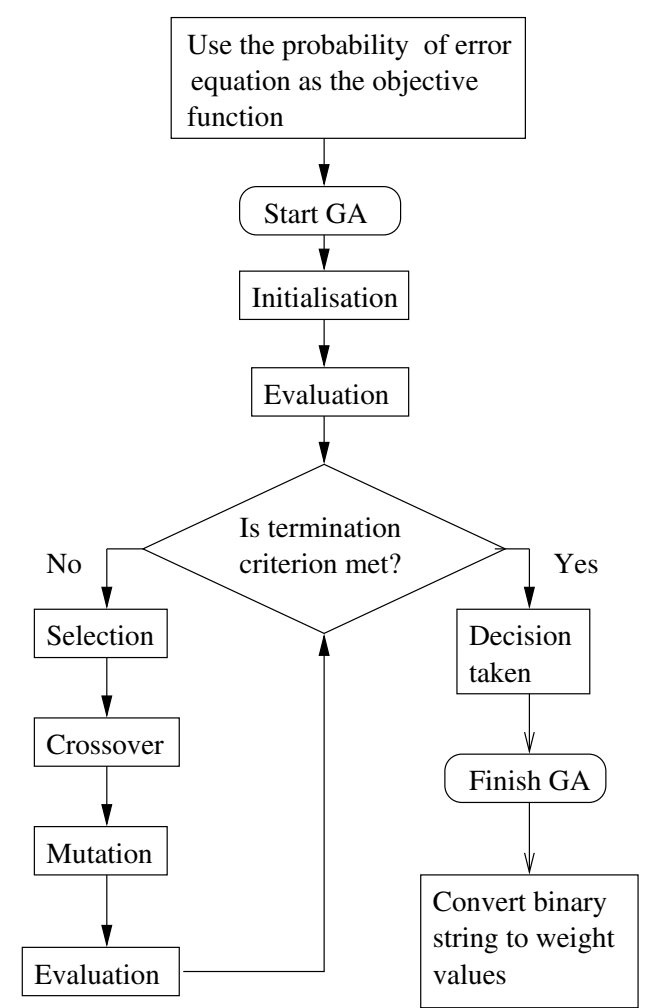

Figure 2: Flowchart for the probability error optimisation using GA.

\section{GENETIC ALGORITHM}

Even though the MBER detector of [7] is capable of maintaining a good performance, the convergence of the algorithm is sensitive to the choice of the algorithm's parameters. For example, the choice of the initial condition for the MBER MUD is critical in order for the solution to converge to the minimum of the BER surface. In [7], the MMSE SDMA MUD weight solution has been used for initialising the CG algorithm based MUD. However, this choice of initial conditions does not necessarily guide the algorithm's convergence to the required MBER solution. Another parameter that affects the performance of the MBER detector is the step size $\mu$ used for updating the array weights in the direction opposite to the BER gradient. The choice of this step size must be based on a compromise, since a step-size that is too high might not allow convergence to the minimum BER point, whereas the opposite scenario will require a high number of iterations for attaining convergence to the MBER solution. An attractive method that might be able to assist the MBER MUD in circumventing the above-mention problems is constituted by the family of genetic algorithms (GA) [8, 9].

Although GAs have been used in numerous applications, such as machine learning and modelling adaptive processes, by far the largest application of GAs is in the domain of function optimisation $[9,10]$. GAs are different from traditional algorithms, because they do not attempt to optimise the desired decision variable [9]. Instead, they encode the decision variables such as for example the SDMA MUD's weight vec-

\begin{tabular}{|l|r|}
\hline Parameter & Value or type \\
\hline \hline SDMA & 4 \\
\hline Number of users & 4 \\
\hline Receiver antennas & 128 \\
\hline OFDM & 32 \\
\hline Number of subcarriers & \\
\hline Length of cyclic prefix & Non-overlapping \\
\hline GA & 30 \\
\hline GA type & 100 \\
\hline Population size & Flip mutator \\
\hline Number of generations & 0.01 \\
\hline Mutation type & Binary string \\
\hline Probability of mutation & Uniform \\
\hline Crossover type & Single-point crossover \\
\hline Probability of crossover & Bit comparator \\
\hline Genome type & Roulette wheel \\
\hline Initialization & Exact MBER \\
\hline Comparison & Gaussian channel [1] \\
\hline Encoding/decoding & Binary encoding and decoding \\
\hline Selection & $+0.2881^{-11}$ dispersive \\
\hline Others & \\
\hline MBER & \\
\hline $\begin{array}{l}\text { Channel impulse } \\
\text { response }\end{array}$ & \\
\hline
\end{tabular}

Table 1: Parameters for the GA simulations.

tors into finite-length strings or GA individuals, which are then optimised. In the case of SDMA-MUDs, both the real and imaginary part of a complex-valued weight have to be represented by a single GA string to create an individual. A GA does not commence its optimisation process from a single point in the search space, but rather from an entire set of individuals, which form the initial population. In other words, GAs may be invoked in robust global search and optimisation procedures that do not require the knowledge of the objective function's derivatives or any gradient-related information concerning the search space. Hence, non-differentiable functions as well as functions with multiple local minima, like the BER surface of the SDMA-MUD, represent classes of problems, where GAs can be efficiently applied. For further details on the origin of GAs and its applications, the readers are referred to the impressive compilation of ideas in [9-11].

\section{SIMULATION RESULTS}

In this contribution, a GA is used for finding the best SDMAMUD weight vectors that will minise the probability of error in Eq. 5. The flowchart of the GA process is shown in Fig. 2. Firstly, the probability of error is used as the objective function to be solved by the GA. Each individual in a population represents either the real or imaginary part of the SDMA-MUD weight values. At the beginning of the GA process the individuals are initialised randomly. These individuals are then evaluated for the sake of finding the best array-weights in the 


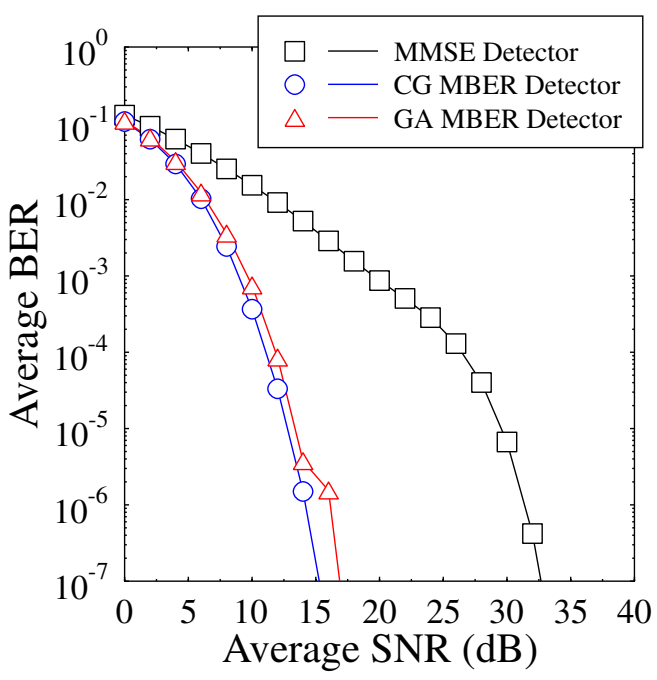

(a) User 1

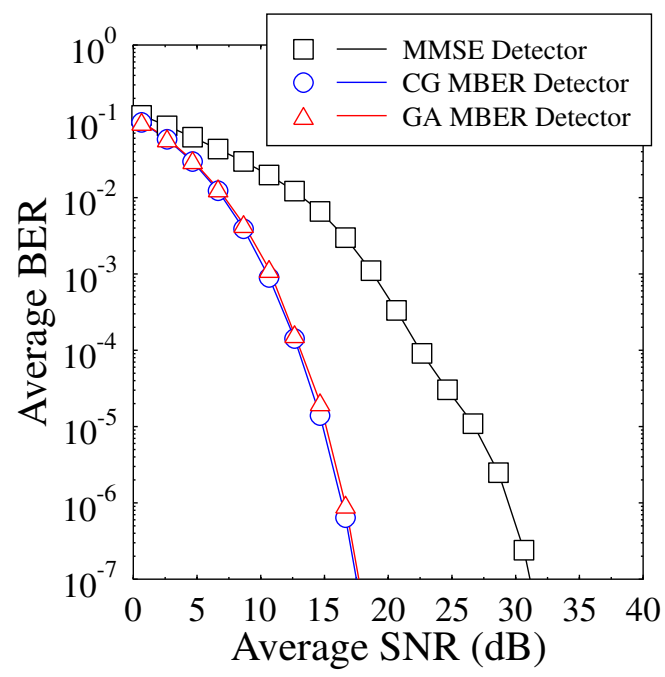

(c) User 3

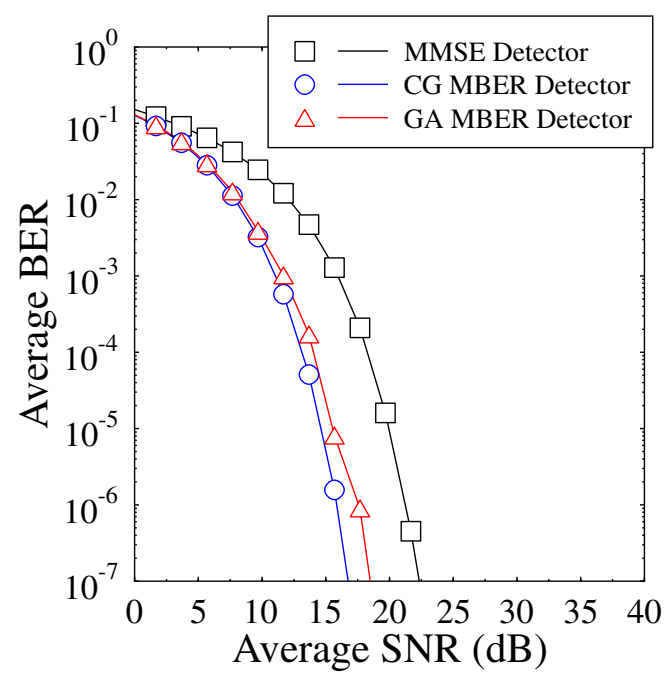

(b) User 2

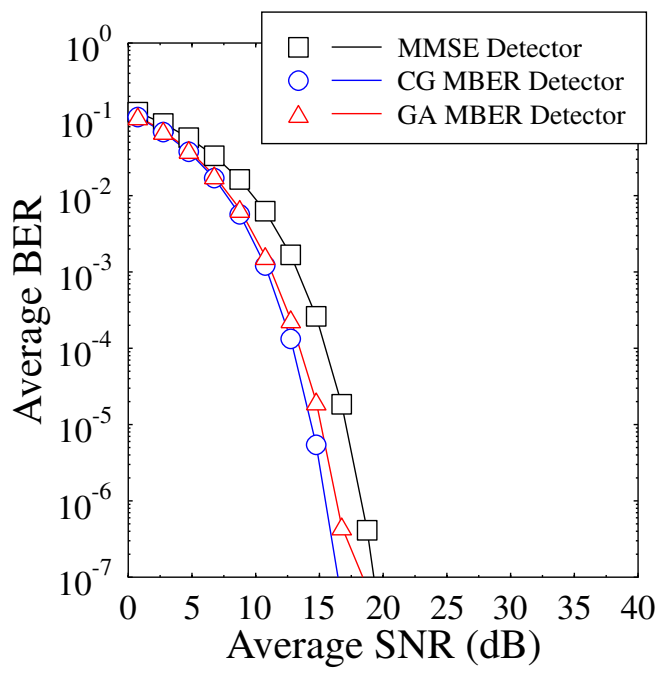

(d) User 4

Figure 3: The BER performance of the four different users in an SDMA system employing four receiver antennas and 128subcarrier OFDM for communicating over the OFDM symbol-invariant dispersive Gaussian channel given in Table 1.

sense of the MBER-related objective function. This GA operation is followed by the selection, crossover and mutation processes, before the individuals are re-evaluated again. The process will continue, until the specific termination criteria advocated is satisfied.

The parameters used for our simulations are outlined in Table 1. The channel that we used in the simulation is the dispersive Gaussian channel, where the $z$-domain transfer function associated with the channel impulse response is given by $h(z)=0.8854+0.3504 z^{-6}+0.2881 z^{-11}[1]$. As a starting point, we used binary type genomes [9] for representing the GA's individuals. Therefore each real and imaginary part of the weight vectors is represented by a 16-bit binary string.
The GA's termination criterion is constituted by the maximum affordable number of generations. Following the termination process, the best individual encountered is deemed to be the best MBER weight solution and hence it is converted to the real and imaginary values of the weight vectors. Our prelimiary results derived for four different users employing the parameters summarised in Table 1 are presented in Fig. 3. We can see from the figure that the MMSE result for each user varies due to the difference in the CIR received at the base station antennas.

\subsection{Complexity comparison}

The advantage of using GAs compared to the CG method [7] for determining the MBER MUD's weight values is that the 


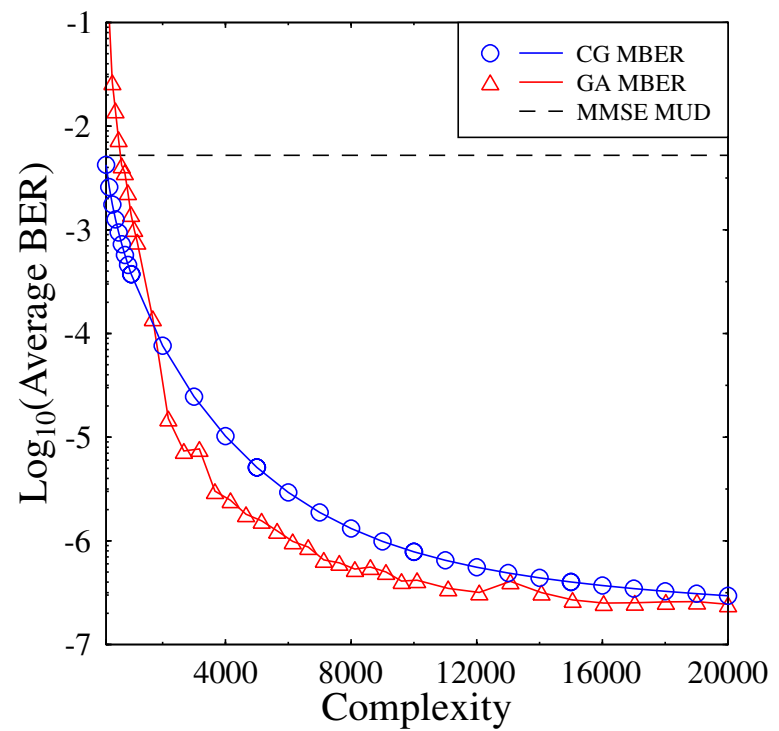

Figure 4: The BER performance of User 1 versus complexity for the GA and CG MBER MUD invoked in the OFDM/SDMA system employing $\mathrm{P}=4, \mathrm{~L}=4$ and 128-subcarrier OFDM for communicating over the symbolinvariant dispersive Gaussian channel given in Table 1 at $\mathrm{SNR}=15 \mathrm{~dB}$. The complexity calculations were described in Sec. 4.1.

GA does not neccessarily require a good initial weight guess for exhibiting rapid convergence. In this section, we will investigate the complexity of the two methods.

The complexity of the CG algorithm is proportional to the number of iterations used for finding the MBER solution on the BER surface. In each iteration the gradient of Eq. 7 will have to be calculated and the SDMA-MUD weight values will be updated accordingly. Therefore the complexity of the CG method can be estimated as:

$$
\operatorname{Compl}\{C G\} \simeq \text { Maximum number of iterations. }
$$

On the other hand, if we used the maximum number of generations as the termination criterion in the GA, each generation of the population contains a certain number of individuals, thus the complexity of the GA aided MUD is proportional to the product of the population size and the number of generations used, which is given by:

$$
\operatorname{Compl}\{G A\} \simeq \text { Population size } \times \text { Generation. }
$$

By using Eq. 8 and Eq. 9, we can compare the complexity of the two methods. Fig. 4 shows the probability of error for User 1 at $\mathrm{SNR}=15 \mathrm{~dB}$ for the $\mathrm{P}=4$ and $\mathrm{L}=4$ system configuration. We can see from the figure that the GA-aided SDMA-MUD will reach the minimum BER at a lower complexity compared to the CG method.

\section{CONCLUSION}

In this contribution, we have shown that GAs may be applied in the context of an SDMA OFDM system for determining the
MBER MUD's weight vectors. The GA-aided system has an edge over the CG-based system, because it does not require an initial weight solution. It was also shown that the GA is capable of approaching the MBER solution at a lower complexity than the CG algorithm. Our future work will invoke forward error correction codes in high-dimensional SDMA-MUDs.

\section{REFERENCES}

[1] L. Hanzo, M. Münster, B. J. Choi and T. Keller, OFDM and MC-CDMA. West Sussex, England: John Wiley and IEEE Press, 2003.

[2] P. Vandenameele, L. van Der Perre, and M. Engels, Space Division Multiple Access for Wireless Local Area Networks. Boston: Kluwer Academic Publishers, 2001.

[3] R. C. de Lamare and R. Sampaio-Neto, "Adaptive MBER Decision Feedback Multiuser Receivers in Frequency Selective Fading Channels," IEEE Communications Letters, vol. 7, no. 2, pp. 73-75, February 2003.

[4] C.-C. Yeh and J. R. Barry, "Adaptive Minimum Bit-Error Rate Equalization for Binary Signalling," IEEE Transactions on Communications, vol. 48, no. 7, pp. 1226-1235, July 2000 .

[5] S. Chen, A. K. Samingan, B. Mulgrew, and L. Hanzo, "Adaptive Minimum-BER Linear Multiuser Detection for DS-CDMA Signals in Multipath Channels," IEEE Transactions on Signal Processing, vol. 49, no. 6, pp. 1240-1247, June 2001.

[6] D. Gesbert, "Robust Linear MIMO Receivers: A Minimum Error-Rate Approach," IEEE Transactions on Signal Processing, vol. 51, no. 11, pp. 2863-2871, November 2003.

[7] M. Y. Alias, A. K. Samingan, S. Chen, and L. Hanzo, "Multiple Antenna Aided OFDM Employing Minimum Bit Error Rate Multiuser Detection," IEE Electronics Letters, vol. 39, no. 24, pp. 1769-1770, 27 November 2003.

[8] L. Hanzo, L-L. Yang, E-L. Kuan and K. Yen, Single- and Multi-Carrier DS-CDMA: Multi-User Detection, SpaceTime Spreading, Synchronisation, Networking and Standards. West Sussex, England: John Wiley and Sons, 2002.

[9] D. E. Goldberg, Genetic Algorithms in Search, Optimization, and Machine Learning. Reading, Massachusetts: Addison-Wesley, 1989.

[10] J. Holland, Adaptation in Natural and Artificial Systems. Ann Arbor, Michigan: University of Michigan Press, 1975.

[11] M. Mitchell, An Introduction to Genetic Algorithm. Cambridge, Massachusetts: MIT Press, 1996. 